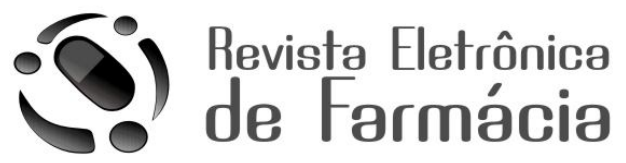

REF - ISSN 1808-0804 Vol. VII (3), 01 - 15, 2010

\title{
ALVOS MOLECULARES UTILIZADOS EM PCR PARA O DIAGNÓSTICO DA LEISHMANIOSE VISCERAL HUMANA
}

\section{MOLECULAR TARGETS USED IN PCR FOR THE DIAGNOSIS OF HUMAN VISCERAL LEISHMANI ASIS}

\section{Maria Almerice Lopes da Silva, Rafael Acioli Medeiros, Sinval Brandão-Filho, Fábio Lopes de Melo, Zulma Medeiros}

E-mail da autora para correspondência: almerice_lopes@yahoo.com.br

Recebido em 10/ 06/ 2010, Aceito em 29/ 07/ 2010

RESUMO: A dificuldade do diagnóstico do calazar humano traz a necessidade do emprego de ferramentas sensíveis e específicas. Nesse contexto, as abordagens em PCR vem se destacando, com uma ampla gama de alvos e iniciadores já padronizados. Porém, há uma carência de dados sistematizados que apontem qual o melhor alvo e iniciadores a serem utilizados em determinadas regiões e em situações específicas. Assim, este trabalho almejou descrever as informações disponíveis na literatura para auxiliar na escolha dos sistemas de PCR pelos profissionais de saúde na rotina dos serviços de laboratório. A identificação dos trabalhos foi feita por busca em bases de dados, que após leitura dos resumos/abstracts foram inclusos nessa revisão. O artigo aponta quais as vantagens e desvantagens dos alvos moleculares e iniciadores, oferecendo informações para a escolha dos alvos e iniciadores adequados segundo a necessidade do serviço pelos profissionais.

Palavras-chave: diagnóstico, leishmaniose visceral, reação em cadeia da polimerase, saúde pública.

ABSTRACT: The difficulty for diagnosing human leishmaniasis brings the need of using sensitive and specific tools. In this context, PCR approaches have been increasing, with a wide range of targets and primers already standardized. However, there is a lack of standardized data that suggest what is the best target and primers to use in certain regions and in specific situations. Thus, this work aims at describing the information available in the literature to assist in the selection of PCR systems for health professionals in routine laboratory services. The identification of the papers was provided by research in databases; after the reading of summaries the exemplars selected were included in this review. The article points out the advantages and disadvantages of targets and primers, providing information for the choice of appropriate targets and primers according to the needs of the work developed by the professionals.

Keywords: diagnosis, visceral leishmaniasis, polymerase chain reaction, public health. 
Silva, M. A. L; Medeiros, R. A.; Filho, S. B.; Melo, L. F. Medeiros, Z./Revista Eletrônica de Farmácia

Vol 7 (3), $01-15,2010$

\section{NTRODUÇÃO}

A leishmaniose visceral (LV), ou calazar, é uma doença sistêmica que pode ser fatal se não tratada. No continente americano, o Brasil apresenta a maior endemicidade, com cerca de $97 \%$ de todos os casos americanos. A região Nordeste brasileira possui $82,5 \%$ das notificações brasileiras (MAIA-ELKHOURY et al., 2008).

No diagnóstico laboratorial, a demonstração de formas amastigotas no aspirado de medula óssea, baço, linfonodos ou fígado é considerado o padrão ouro. Porém, são procedimentos invasivos, possuem sensibilidade variável e contraindicações. Em relação aos testes sorológicos disponíveis a (imunofluorescência indireta (IFI), teste de aglutinação direta (DAT) e a imunocromatografia utilizando o antígeno rK39) possuem limitações, tanto na sensibilidade quanto na especificidade (SANDAR; RAIA, 2002; GONTIJO; MELO, 2004; DOURADO et al., 2007). A pesquisa de antígenos em urina pela aglutinação em látex (KAtex) tem sido apontada como uma ferramenta promissora, mesmo nas difíceis condições de campo (ATTAR et al., 2001; GARCÍA-GARCÍA et al., 2006; KALLEL et al., 2007; GAVGANI; KHADEMVATAN; GHAZANCHAEI, 2008).

A PCR tem sido vista como um diagnóstico alternativo quando o paciente apresenta como hipótese diagnóstica calazar ou coinfecção e microscopia e sorologia são negativas ou indeterminadas (ALAM et al., 2009). Os ensaios já foram padronizadas com diversos fluidos corporais, como sangue, aspirado de medula óssea, soro e urina (ANDRESEN et al., 1997; FISA et al., 2008a). Vários sistemas baseados em PCR têm sido desenvolvidos para Leishmania, e muitas sequências alvo já foram identificadas, como também um grande número de oligonucleotídeos iniciadores específicos (primers) desenhados.

Assim, o objetivo dessa revisão é sistematizar as informações, auxiliando profissionais e serviços de saúde na implantação dessa ferramenta na rotina de diagnóstico. A identificação dos artigos foi feita por busca nas bases de dados LILACS (Literatura Latino-Americana e do Caribe em Ciências da Saúde), MEDLINE (National Library of Medicine, Estados Unidos), PUBMED (National Institutes of Health) e SCOPUS, reunindo publicações em inglês, espanhol e português no período de 1999 a 2009. Os descritores utilizados foram: "leishmaniose visceral" ou "visceral leishmaniasis" e "PCR". Foram excluídos os trabalhos que não objetivassem o diagnóstico por PCR para LV humana. Assim, a pesquisa resultou em 269 artigos. Após leitura de seus abstracts/resumos, 84 artigos foram selecionados para a elaboração deste artigo.

$$
\text { Os iniciadores citados foram }
$$
submetidos à análise de homologia utilizandose o servidor primer-BLAST 2.0 ("Basic Local Alignment Search Tool") do National Center for Biotechnology Information (NCBI) da biblioteca Nacional de Medicina do $\mathrm{NIH}$ (National Institute of Health), Maryland, EUA. No quadro 1 são apresentados os iniciadores, com respectivas citações bibliográficas, seus 
Silva, M. A. L; Medeiros, R. A.; Filho, S. B.; Melo, L. F. Medeiros, Z./Revista Eletrônica de Farmácia Vol 7 (3), $01-15,2010$

alvos moleculares e primer-BLAST, que são

citados

neste

trabalho.

Quadro 1 - Alvos moleculares e iniciadores estudados para o diagnóstico da LV humana

\begin{tabular}{|c|c|c|c|}
\hline I niciadores & Autores & $\begin{array}{c}\text { Alvo } \\
\text { molecular }\end{array}$ & $\begin{array}{c}\text { Análise pela } \\
\text { ferramenta } \\
\text { primer-BLAST }\end{array}$ \\
\hline $\begin{array}{l}\text { RV1: 5'-CTTTTCTGGTCCCGCGGGTAGG- } \\
\text { 3' } \\
\text { RV2: 5'-CACCTGGCCTATITACACCA-3' }\end{array}$ & $\begin{array}{l}\text { LE FICHOUX et } \\
\text { al. (1999) }\end{array}$ & $\begin{array}{l}\text { Minicírculo do } \\
\text { DNA do } \\
\text { cinetoplasto } \\
\text { (kDNA) }\end{array}$ & $\begin{array}{l}\text { L. infantum, L. } \\
\text { donovani e L. } \\
\text { major. }\end{array}$ \\
\hline $\begin{array}{l}\text { AJ S31: } \\
\text { 5'-GGGGTTGGTGTAAAATAGGGCCGG-3' } \\
\text { DeBY: 5'-CCAGGTTCCCGCCCCGGAG-3' }\end{array}$ & $\begin{array}{l}\text { SMYTH et al. } \\
\text { (1992) }\end{array}$ & & Leishmania SP \\
\hline $\begin{array}{l}\text { Ldl: } \\
\text { 5'-AAATCGGCTCCGAGGCGGGAAAC-3' e } \\
\text { 5'-GGTACACTCTATCAGTAGCAC-3' }\end{array}$ & $\begin{array}{l}\text { SALOTRA et al. } \\
\qquad(2001)\end{array}$ & & L. donovani \\
\hline $\begin{array}{l}\text { MC1: 5'-GTTAGCCGATGGTGGTCTTG-3' } \\
\text { MC2: 5'-CACCCATTTTTCCGATITTG-3' }\end{array}$ & $\begin{array}{l}\text { CORTES et al. } \\
(2004)\end{array}$ & & $\begin{array}{l}\text { L. infantum, L. } \\
\text { chagasi, L. } \\
\text { donovani e } \\
\text { Caenorhabditis } \\
\text { elegans }\end{array}$ \\
\hline $\begin{array}{l}\text { R221: 5'-GGTTCCTTTCCTGATTTACG-3' } \\
\text { R332: 5'-GGCCGGTAAAGGCCGAATAG-3' } \\
\text { R223: 5'-TCCCATCGCAACCTCGGTT-3' } \\
\text { R333: 5'-AAAGCGGGCGCGGTGCTG-3' }\end{array}$ & $\begin{array}{l}\text { VAN EYS et al. } \\
\text { (1992) }\end{array}$ & $\begin{array}{l}\text { Subunidade } \\
\text { menor do } \\
\text { ribossomo } \\
\text { (SSUrRNA) }\end{array}$ & $\begin{array}{l}\text { Trypanosomatidae } \\
\text { sp }\end{array}$ \\
\hline $\begin{array}{l}\text { 18S-L-F: } \\
\text { 5'-CGTAGTTGAACTGTGGGCTGTGC-3' } \\
\text { 18S-L-R: } \\
\text { 5'-ACTCCCGTGTTCTTGTTTCTTTGAA-3' }\end{array}$ & $\begin{array}{l}\text { DEBORGGRAEVE } \\
\text { et al. (2008) }\end{array}$ & & $\begin{array}{l}\text { Trypanosomatidae } \\
\text { sp }\end{array}$ \\
\hline $\begin{array}{l}\text { LITSR: 5'-CTGGATCATTTTCCGATG-3' } \\
\text { L5.8S: 5'- TGATACCACTTATCGCACTT-3' } \\
\text { LITSV: 5'-ACACTCAGGTCTGTAAAC-3' } \\
\text { L5.8SR: 5'-AAGTGCGATAAGTGGTA-3' }\end{array}$ & $\begin{array}{l}\text { EL TAIL et al. } \\
(2000)\end{array}$ & $\begin{array}{l}\text { Espaçador } \\
\text { Transcrito } \\
\text { Interno (ITS) }\end{array}$ & $\begin{array}{l}\text { Trypanosomatidae } \\
\text { sp }\end{array}$ \\
\hline $\begin{array}{l}\text { Hsp70sen: } \\
\text { 5'-GACGGTGCCTGCCTACTTCAA-3' } \\
\text { Hsp70ant: } \\
\text { 5'-CCGCCCATGCTCTGGTACATC-3' }\end{array}$ & $\begin{array}{l}\text { GARCIA et al. } \\
\quad(2004)\end{array}$ & Gene HSP70 & $\begin{array}{l}\text { Leishmania sp, } \\
\text { Trypanosoma sp. }\end{array}$ \\
\hline $\begin{array}{l}\text { LDS: 5'-GCGACGACAAGCCCATGATT-3' } \\
\text { LDK: 5'- GCGTCGGCTCGTTGATGATG-3' }\end{array}$ & $\begin{array}{l}\text { ARORA et al. } \\
\quad(2008)\end{array}$ & & $\begin{array}{c}\text { Trypanosomatidae } \\
\text { sp. }\end{array}$ \\
\hline $\begin{array}{l}\text { SG1: } \\
\text { 5'-GTCTCCACCGAGGACCTCACCGA-3' } \\
\text { SG2: } \\
\text { 5'-TGATGTAGCCGCCCTCCTCGAAG-3' }\end{array}$ & $\begin{array}{l}\text { GUERBOUJ et al. } \\
(2001)\end{array}$ & Gene gp63 & $\begin{array}{l}\text { Leishmania SP, } \\
\text { Crithidia } \\
\text { fasciculata, } \\
\text { Azotobacter } \\
\text { vinelandii, } \\
\text { Pseudomonas } \\
\text { stutzeri, } \\
\text { Micromonas sp., }\end{array}$ \\
\hline
\end{tabular}


Silva, M. A. L, Medeiros, R. A.; Filho, S. B.; Melo, L. F. Medeiros, Z./Revista Eletrônica de Farmácia Vol 7 (3) , $01-15,2010$

\begin{tabular}{|c|c|c|c|}
\hline $\begin{array}{l}\text { PDD1: } \\
\text { 5'-TCGGTGAGGTCCTCGGTGGAGAC-3' } \\
\text { PDD2: } \\
\text { 5'-CTTCGAGGAGGGCGGCTACATCA-3' }\end{array}$ & & & $\begin{array}{l}\text { L. infantum, L. } \\
\text { braziliense, } \\
\text { Burkholderia } \\
\text { gluma }\end{array}$ \\
\hline $\begin{array}{l}\text { TV255: } \\
\text { 5'-AGTACGGCTGCGACACCTTGGAG-3' } \\
\text { TV256: } \\
\text { 5'-GTTCCGGCCCCACGGCATCACC-3' }\end{array}$ & $\begin{array}{l}\text { TUPPERWAR et } \\
\text { al. (2008) }\end{array}$ & & $\begin{array}{l}\text { Leishmania sp, } \\
\text { Xylanimonas } \\
\text { cellulosilytica, } \\
\text { Corynebacterium } \\
\text { efficiens }\end{array}$ \\
\hline $\begin{array}{l}\text { K26f: 5'-ACGAAGGACTCCGCAAAG-3' } \\
\text { K26r: 5'-TTCCCATCGTTTTGCTG-3' }\end{array}$ & $\begin{array}{l}\text { HARALAMBOUS } \\
\text { et al. (2008) }\end{array}$ & Gene K26 & $\begin{array}{l}\text { Complexo L. } \\
\text { donovani }\end{array}$ \\
\hline $\begin{array}{l}\text { Ext: 5'-AATTCGACGATCACGAGGTC-3' } \\
\text { E2b: 5'-CGACTCGGTTGGCACACTG-3' }\end{array}$ & $\begin{array}{l}\text { FISA et al. } \\
\text { (2008b) }\end{array}$ & $\begin{array}{l}\text { Fragmentos } \\
\text { gênicos }\end{array}$ & L. infantum \\
\hline $\begin{array}{l}\text { P-l: 5'-ACGAGGTCAGCTCCACTCC-3' } \\
\text { P-2: 5'- CTGCAACGCCTGTGTCTA CG-3' }\end{array}$ & $\begin{array}{l}\text { PIARROUX et al. } \\
\text { (1993) }\end{array}$ & & L. infantum \\
\hline $\begin{array}{l}\text { Fme: 5'-TATTGGTATGCGAAACTTCCG-3' } \\
\text { Rme: 5'-CAGAAACTGATACTTATATAGCG- } \\
\text { 3' }\end{array}$ & $\begin{array}{l}\text { MARFURT et al. } \\
\text { (2003a) }\end{array}$ & $\begin{array}{l}\text { Gene do mini- } \\
\quad \text { exon }\end{array}$ & $\begin{array}{l}\text { Trypanosomatidae } \\
\text { sp. }\end{array}$ \\
\hline $\begin{array}{l}\text { S-1629: } \\
\text { 5'-gggaattCAATAT/AAGTACAGAAACTG } \\
\text { S-1630: } \\
\text { 5'- gggaagcTTCTGTACTT/ATATTGGTA }\end{array}$ & $\begin{array}{l}\text { KATAKURA et al. } \\
(1999)\end{array}$ & & Múltiplos alvos \\
\hline
\end{tabular}

\section{ALVOS MOLECULARES E I NI CI ADORES}

Os ensaios baseados em PCR constituem atualmente a principal abordagem no diagnóstico molecular. Além da pequena quantidade necessária de material biológico, outro ponto importante da PCR é a diferenciação dos agentes infecciosos, que pode ser feita pela análise de polimorfismo dos alvos (BHATTARAl et al., 2009). Os alvos são importantes pelas suas sensibilidade e especificidade, características do ensaio que são intrínsecas a escolha dos iniciadores, que podem resultar falso positivos ou falso negativos (DENIAU et al., 2003).

\section{Minicírculo do cinetoplasto}

O DNA do cinetoplasto ( $k D N A$, do inglês kinetoplast) representa $20-25 \%$ do DNA do parasito e consiste em uma rede de moléculas circulares, concatenadas, divididas em maxicírculos e minicírculos (TELLERIA et al., 2006). São cerca de 50 maxicírculos (com 20.000 a 35.000 pb (pares de base)), que contêm genes que codificam proteínas mitocondriais e o RNA ribossomal (rRNA), enquanto os minicírculos são no número de 10.000 a 20.000, com sequências de 500 a $2.500 \mathrm{pb}$ (CORTES, 2008). Diante dos alvos que serão descritos nesta revisão, pode-se dizer que o minicírculo kDNA é o alvo mais 
Silva, M. A. L, Medeiros, R. A.; Filho, S. B.; Melo, L. F. Medeiros, Z./Revista Eletrônica de Farmácia Vol 7 (3), $01-15,2010$

estudado e aplicado nas pesquisas moleculares para o diagnóstico de calazar humano. Sua vantagem está no fato do grande número de cópias por célula.

Os iniciadores desenhados por Le Fichoux et al. (1999) foram os mais aplicados na amplificação do minicírculo do kDNA (Quadro 1). A região amplificada é a LT1, que apresenta uma sequência de 145 pb, e os sistemas desenvolvidos já foram aplicados com sucesso na identificação de Leishmania em amostras de medula, sangue, soro e urina, de pacientes imunocompetentes e imunocomprometidos (ATTAR et al., 2001; FISSORE et al., 2004; MARY et al., 2004, 2006; KONGKAEW et al., 2007; FAKHAR et al., 2008; MOTAZEDIAN et al., 2008; JUNIOR et al., 2009). Os bons resultados para esses iniciadores foram obtidos tanto utilizando técnicas convencionais de PCR como a PCR em Tempo Real (MARY et al., 2004; GERAMIZADEH; FAKHAR; MOTAZEDIAN, 2006; KONGKAEW et al., 2007; SUKMEE et al., 2008; VILLINSKIA et al., 2008). Os sistemas apresentaram valores de detecção de 0,001 parasito/mL, identificando DNA de Leishmania em portadores assintomáticos, quando as técnicas convencionais de diagnóstico falharam, indicando uma boa correlação entre os níveis de parasitemia e o estado clínico dos pacientes (MARY et al., 2004; FAKHAR et al., 2008). Porém, sistemas que produzem amplicons com baixo número de $\mathrm{pb}$ podem apresentar erros na interpretação dos resultados, devido ao tamanho próximo ao dos dímeros de iniciadores.
O par de primers AJS31/DeBY (SMYTH et al., 1992) produz um fragmento de $805 \mathrm{pb}$ do kDNA para L. infantum e L. donovani, e 780 pb para L. chagasi (LAMBSON; SMYTH; BARKER, 1999, 2000; MONROY-OSTRIA; HERNANDEZ-MONTES; BARKER, 2000; MORALES et al., 2001; RODRIGUEZ et al., 2005; BOTILDE et al., 2006). Ao se aplicar enzimas de restrições, Morales et al., 2002, distinguiram entre recidivas e reinfecções póstratamento na coinfecção por HIV/Leishmania. Resultados significativos também foram obtidos com o uso dos iniciadores Ld I (SALOTRA et al., 2001), que amplificam um fragmento de aproximadamente $600 \mathrm{pb}$, com uma detecção de até $1 \mathrm{fg}$ de DNA de L. donovani (SREENIVAS et al., 2004; MAURYA et al., 2005; SHARMA et al., 2010). A especificidade dos iniciadores foi avaliada com DNA de L. major, L. tropica, Plasmodium spp., Mycobacterium leprae, Mycobacterium tuberculosis, onde não se observou amplificação (SALOTRA et al., 2001). Em relação aos iniciadores $\mathrm{MC1} / \mathrm{MC2}$, o produto da amplificação é uma banda de 447 pb (CORTES et al., 2004). Diante dos ensaios com amostras biológicas, esses iniciadores mostraram-se específicos para as espécies L. donovani e L. infantum (CORTES et al., 2004, 2006; ROLÃO et al., 2004).

Muitos outros iniciadores foram desenvolvidos nesta última década para pesquisa de DNA de Leishmania utilizando como alvo o kDNA (BRENIÈRE et al., 1999; MARTIN-SANCHEZ et al., 2001; ANDERS et al., 2002; KYRIAKOU et al., 2003; MARFURT et al., 2003a; DISCH et al., 2006; BRUSTOLONI et al., 2007; ASSIS et al., 
Silva, M. A. L, Medeiros, R. A.; Filho, S. B.; Melo, L. F. Medeiros, Z./Revista Eletrônica de Farmácia Vol 7 (3), $01-15,2010$

2009). Esse alvo possui uma considerável heterogeneidade o que pode acarretar em perda da acurácia do ensaio, o que não é observado em outros alvos moleculares (MARY et al., 2004). Na aplicação de enzimas de restrição ao kDNA (PCR-RFLP), essa heterogeneidade dificulta a construção de padrões por RFLP (Restriction Fragment Length Polymorphism) devido ao grande número de fragmentos restritos, restringindo a utilização dos iniciadores entre isolados próximos ou apenas permite a identificação de grupos ou isolados de mesma área.

\section{DNA ribossômico}

O DNA ribossômico (rDNA) possui unidades repetidas ou em tandem dentro da região nucleolar. Nas células eucarióticas, há cerca de 100 a 500 cópias do gene rDNA no genoma nuclear, onde cada unidade de transcrição, é composta de uma região promotora líder, o espaçador transcrito externo (ETS - External Transcribed Spacer), uma região codificadora do rRNA 18S, um espaçador não codificante interno (ITS-1), uma região codificadora de rRNA 5,8S, um outro espaçador não codificante interno (ITS2), uma região codificadora de rRNA $28 S$ e, finalmente, um segmento intergênico espaçador não transcrito, IGS (MATEUS et al., 2006).

Apesar do baixo número de cópias do rDNA em comparação ao kDNA, suas subunidades são bem exploradas no desenvolvimento de ferramentas moleculares para diagnóstico da infecção por Leishmania, sendo esse alvo um dos mais explorados na identificação de espécies.

\subsection{Subunidade menor do ribossomo}

Os genes codificantes do RNA presente na subunidade menor do ribossomo (SSUrRNA) apresentam múltiplas cópias e são bem conservados para o gênero em estudo, permitindo o desenho dos iniciadores. Esse alvo não apresenta $\mathrm{o}$ problema de heterogeneidade do kDNA, podendo a informação discriminatória do segmento variável ser acessada por sequenciamento ou por hibridação, produzindo resultados positivos pela PCR, independente da linhagem, grupo ou espécie (FLOETER-WINTER, 2010). Uma desvantagem na aplicação desse alvo é que relações filogenéticas não puderam ser esclarecidas (ULIANA et al., 1991).

Os iniciadores R221, R332, R223 e R333 apresentados por Van Eys et al. (1992) são os mais descritos na amplificação de regiões do SSU-rRNA, produzindo fragmentos de 600-650 pb dependendo da espécie de Leishmania (SCHÖNIAN et al., 2003). Os estudos apontam um desempenho satisfatório desses iniciadores nas PCRs desenvolvidas, ao serem comparados com a pesquisa direta do parasito e sorologia (PIZZUTO et al., 2001; DONCKER et al., 2005; STARK et al., 2006; ANTINORI et al., 2007; SALAM et al., 2009), assim como no monitoramento pósterapêutico de pacientes imunocomprometidos (LACHAUD et al., 2000, 2001). A Nested-PCR utilizando os pares R221/R332 na primeira reação e R223/R333 na segunda reação detectou 0,01 promastigota (CRUZ et al., 2002). Esta abordagem foi superior às técnicas convencionais em pacientes coinfectados por HIV/Leishmania (CRUZ et al., 
Silva, M. A. L, Medeiros, R. A.; Filho, S. B.; Melo, L. F. Medeiros, Z./Revista Eletrônica de Farmácia Vol 7 (3), $01-15,2010$

2002) e em crianças imunocompetentes (CRUZ et al., 2006).

Recentemente, Deborggraeve et al. (2008), desenharam os iniciadores senso 18SL-F e antissenso 18S-L-R, que reproduzem um fragmento de $115 \mathrm{pb}$ do gene 18S rRNA. A PCR apresentou uma detecção de até $10 \mathrm{fg}$ de DNA, não sendo observadas reações cruzadas com Plasmodium falciparum, Mycobacterium tuberculosis, Schistosoma mansoni, Trypanossoma brucei gambiense e Trypanossoma cruzi. Bhattarai et al. (2010) aplicaram esse sistema na investigação de pacientes assintomáticos que moravam no mesmo domicílio de casos confirmados de calazar, alcançando um excelente desempenho da ferramenta molecular em comparação ao DAT no diagnóstico da infecção por Leishmania.

$\mathrm{Na}$ análise pela ferramenta primerBLAST, todos os iniciadores citados para o SSUrRNA podem também amplificar esse alvo em outros tripanossomatídeos, produzindo bandas de tamanho similar aos amplificados com o gênero Leishmania. Tal fato pode indicar um problema no uso desse ensaio em áreas coendêmicas para esses parasitos, podendo resultar, por exemplo, em reação cruzada com doença de Chagas.

\subsection{Espaçador interno transcrito}

O espaçador interno transcrito (ITS) é uma região não codificante encontrada no SSUrRNA, onde o ITS-1 é delimitado pelos genes $18 \mathrm{~S}$ e $5.8 \mathrm{~S}$, enquanto o ITS- 2 pelos genes $5.8 \mathrm{~S}$ e $28 \mathrm{~S}$. Esse espaçador tem sido descrito na identificação de espécies de Leishmania e de suas linhagens por meio da
PCR-RFLP (SCHÖNIAN et al., 2003). Os pares de iniciadores mais utilizados na amplificação da região ITS são LITSR e L5.8S, que reproduz o ITS-1 com produto de 300 - 350 pb, e LITSV e L5.8SR, para o ITS- 2 com produto de 700 pb (EL TAIL et al., 2000; SCHÖNIAN et al., 2003). A ITS-1 PCR apresentou bons resultados em amostra de sangue, aspirado de medula ou esfregaços de lâminas coradas com Giemsa (EL TAIL et al., 2000; BADER et al., 2005; KAZEMI-RAD et al., 2008; ALAM et al., 2009; AMRO et al., 2009). Esses iniciadores parecem ser específicos para o gênero Leishmania, pois não produz bandas frente a DNA de T. cruzi, Escherichia coli, Candida albicans, Tychophywn terrestre e Microsporum audouini (EL TAIL et al., 2000). Porém, utilizando a ferramenta primer-BLAST, observa-se que esses iniciadores podem amplificar regiões de outros tripanossomatídeos, inclusive T. cruzi, com produtos que variam de 300-760 pb de tamanho.

\section{Outras regiões-alvo}

Os microssatélites são loci polimórficos presentes no DNA nuclear amplamente distribuídos nas células eucarióticas e que apresentam sequências simples repetitivas (SSR), composta por 1 a 4 nucleotídeos, que geralmente não excedem 200 pb (BECKMAN; WEBER, 1992). Rossi et al. (1994), identificaram 3 sequências de microssatélites no genoma de Leishmania: (CA)n, (GAC)n e (GGT)n. Segundo Ferreira e Grattapaglia (1998), a utilização dessa região em PCR oferece uma completa cobertura do genoma, sendo úteis para mapeamento genético e 
Silva, M. A. L, Medeiros, R. A.; Filho, S. B.; Melo, L. F. Medeiros, Z./Revista Eletrônica de Farmácia Vol 7 (3), $01-15,2010$

físico de genomas, identificação e discriminação de genótipos e estudos de genética de populações. Botilde et al. (2006) compararam o poder discriminatório em nível de zimodema para L. infantum de quatro métodos moleculares: kDNAPCR-RFLP, cpb e gp63PCR-RFLP, tipagem de microssatélite multilocus (MLMT) e amplificação aleatória do DNA polimórfico (RAPD). O polimorfismo nos microssatélites é simples e facilmente detectado pela PCR, com boa reprodutibilidade e discriminação, podendo ser usado para diferenciar relapsos ou reinfecção em pacientes com múltiplos episódios de LV (BULLE et al., 2002; OCHSENREITHER et al., 2006; KUHLS et al., 2007; SERIDI et al., 2008).

As Heat Shock Proteins (HSPs), ou proteínas do choque térmico, são identificadas como os principais imunógenos em várias doenças infecciosas (ARORA et al., 2008), sendo a família da HSP70 comum em soro de pacientes com LV (WALLACE et al., 1992; ARORA et al., 2000). Após mapeamento de HSP70 CDNA (ARORA; MELBY; SEHGAL, 1995; ARORA et al., 2000), abordagens com PCR foram feitas utilizando os iniciadores Hsp70sen e Hsp70ant (GARCIA et al., 2004). Um produto de $1.300 \mathrm{pb}$ foi amplificado com estes parasitos, o mesmo encontrado com o DNA de T. cruzi, mas nenhum produto foi observado com M. tuberculosis ou Sporothrix schenckii. Dessa forma, falsos positivos podem ser obtidos em pacientes chagásicos.

Arora et al. (2008), desenvolveram uma PCR utilizando os iniciadores desenhados LDS e LDK, amplificando um fragmento de 243 pb do genoma de L. donovani. O ensaio obteve uma sensibilidade de 0,5 pg de DNA, aumentando-se para $0,05 \mathrm{pg}$ ao se utilizar uma sonda interna (583-609 bp) no Southern blot. Os autores não observaram amplificações com DNA das espécies L. infantum, L. tropica, L. major, L. mexicana, L. aethiopica, Entamoeba histolytica, M. tuberculosis e P. vivax. Por ser específico para L. donovani, o uso dessa PCR pode não ser adequado onde outras espécies são os agentes responsáveis pela LV.

A gp63 é a principal glicoproteína presente na membrana celular de Leishmania $\mathrm{sp}$, desempenhando papel fundamental na virulência do parasito e na estimulação da mesma quanto à resposta celular e humoral do hospedeiro (HOYA et al., 1999). Devido ao polimorfismo dessas regiões gênicas, os produtos de amplificação podem ser distintos entre L. infantum e L. donovani, podendo-se aplicar a identificação de espécies e/ou linhagens pela restrição dos fragmentos (ELAMIN et al., 2008). Os iniciadores SG1/SG2, PDD1/PDD2, TV255/TV256 foram desenhados para amplificar regiões codificantes dos genes gp63 (GUERBOUJ et al., 2001; TUPPERWAR et al., 2008). Em relação a parasitos humanos, esses iniciadores parecem específicos para o gênero Leishmania pela análise pelo primer-BLAST.

Outro alvo estudado recentemente é a região gênica que codifica o antígeno K26, uma proteína hidrofílica de superfície. O par de iniciadores K26f/K26r mostrou-se específico para o complexo L. donovani, com produtos que variam de 284-1.300 pb, discriminando entre L. infantum e L. donovani (HARALAMBOUS et al., 2008). 
Silva, M. A. L, Medeiros, R. A.; Filho, S. B.; Melo, L. F. Medeiros, Z./Revista Eletrônica de Farmácia Vol 7 (3), $01-15,2010$

Com o par Fme/Rme (MARFURT et al., 2003a), o produto formado é aproximadamente $400 \mathrm{pb}$ para L. donovani, porém há amplificação de 400-430 pb para outros cinetoplastídeos, como Crithidia bombi, Crithidia lucilia e Crithidia fasciculata, mas nada foi observado para T. b. rhodesiense, T. b. gambiense e T. cruzi.

Fisa et al. (2008b), desenvolveram uma Nested-PCR que tem como iniciadores externos o par Ext/E2b e para segunda reação os iniciadores P-I/P-2 (PIARROUX et al., 1993), produzindo fragmento de 100 pb. A Nested-PCR detectou DNA de Leishmania em $18 \%$ dos pacientes infectados por HIV, cujas culturas foram negativas. Além disso, essa PCR detectou DNA de Leishmania nas amostras biológicas 5 a 6 meses antes de a cultura ser positiva. Quando testada com células mononucleares e com o buffy coat do sangue para transplante, o método molecular foi capaz de identificar um alto número de doadores assintomáticos (SERIN et al., 2005), podendo o sangue ser fonte de infecção para os receptores. Esse sistema pode ser utilizado para monitoramento do tratamento, utilizando como amostras biológicas o sangue ou a urina do paciente (RIERA et al., 2004; FISA et al., 2008a). A análise pelo BLAST demonstrou a especificidade dessa Nested-PCR para L. infantum. Na prática, isso pode dificultar seu uso para diagnóstico de LV por outras espécies do complexo L. donovani. Vale ressaltar o pequeno tamanho da banda produzida (100 $\mathrm{pb})$, o que leva a dificuldade de interpretação de resultados.

Os genes nucleares do mini-exon estão presentes nas células do gênero Leishmania e em outros cinetoplastídeos, mas ausente nos mamíferos e vetores. Estão presentes nessas células em número de cópias de 100-200 tandem, separados por genes transcritos e não transcritos. A região transcrita é composta pelo exon altamente conservado com 39 nucleotídeos, e o intron, moderadamente conservado, variando no tamanho entre as espécies do mesmo gênero ou subgênero (FERNANDES et al., 1994; MARFURT et al., 2003b). Utilizando os iniciadores S-1629 e S1630 para as espécies do complexo L. donovani, todas apresentam um produto de aproximadamente 450 pb (KATAKURA et al., 1999). Bons resultados para diferenciação das espécies em amostras clínicas foram alcançados nas pesquisas onde $\mathrm{o}$ alvo da RFLP-PCR era a região dos mini-exons (MARFURT et al., 2003a, 2003b; SERIN et al., 2005). Ao realizar a análise de homologia pela ferramenta primer-BLAST, verificou-se que esses iniciadores amplificam DNA de uma ampla gama de organismo, inclusive Homo sapiens. Porém, não foi notificado o seu reconhecimento por DNA de Leishmania sp.

\section{CONCLUSÃO}

As abordagens moleculares baseadas em PCR são ferramentas sensíveis e específicas para o diagnóstico da leishmaniose visceral em humanos. Destacamos o poder da PCR em discriminar as espécies de Leishmania, o que não é possível pela visualização microscópica do parasito, pesquisa de antígeno ou pela sorologia. Devido à ampla variedade de alvos e iniciadores disponíveis, ainda não há um procedimento "padrão ouro", o que dificulta a 
Silva, M. A. L, Medeiros, R. A.; Filho, S. B.; Melo, L. F. Medeiros, Z./Revista Eletrônica de Farmácia Vol 7 (3), $01-15,2010$

inserção desse ensaio na rotina dos serviços informações para que o profissional possa de saúde. Nosso trabalho aponta quais as escolher o sistema de PCR mais adequado à vantagens e desvantagens dos principais alvos necessidade do seu serviço.

moleculares e iniciadores, oferecendo

\section{REFERÊNCI AS}

ALAM MZ et al. PCR diagnosis of visceral leishmaniasis in an endemic region, Mymensingh district, Bangladesh. Tropical Medicine International Health, 2009,14(5):499-503.

AMRO A et al. Epidemiology of paediatric visceral leishmaniasis in Hebron district, Palestine. Transaction of the Royal of Society Tropical Medicine and Hygiene, 2009, 103: 731-736.

ANDERS $G$ et al. Distinguishing Leishmania tropica and Leishmania major in the Middle East using the polymerase chain reaction with kinetoplast DNA specific primers. Transaction of the Royal of Society Tropical Medicine and Hygiene, 2002, 96(suppl. 1): 87-92.

ANDRESEN $\mathrm{K}$ et al. Diagnosis of visceral leishmaniasis by the polymerase chain reaction using blood, bone marrow and lymph node samples from patients from the Sudan. Tropical Medicine International Health, 1997, 2(5): 440-444.

ANTINORI $S$ et al. Clinical use of polymerase chain reaction performed on peripheral blood and bone marrow samples for the diagnosis and monitoring of visceral leishmaniasis in HIV-infected and HIV uninfected patients: a single-center, 8-year experience in I taly and review of the literature. Clinical Infectious Diseases, 2007, 44: 1602-1610.

ARORA SK, MELBY PC, SEHGAL S. Lack of serological specificity of recombinant heat shock protein 70 of Leishmania donovani. Immunology and Cell Biology, 1995, (73): 446-451.

ARORA SK et al. Identification of sero-specific epitope of recombinant heat shock protein (HSP70) of Leishmania donovani. J ournal of Parasitic Disease, 2000, 24: 21-26.

. An epitope-specific PCR test for diagnosis of Leishmania donovani infections. Transaction of the Royal of Society Tropical Medicine and Hygiene, 2008, 102: 41-45.

ASSIS TSM et al. Detection of Leishmania kDNA in human serum samples for the diagnosis of visceral leishmaniasis. Transaction of the Royal of Society Tropical Medicine and Hygiene, 2009, 103: 1269-1272.

ATTAR ZJ et al. Latex agglutination test for the detection of urinary antigens in visceral leishmaniasis. Acta Tropical, 2001, 78: (1): 11-16.

BADER KA et al. Short communication: Palestinian infantile visceral leishmaniasis caused by a genetic variant of Leishmania infantum belonging to a new zymodeme. Tropical Medicine and International Health, 2005, 10(6): 618-620.

BECKMAN JS, WEBER J L. Survey of human and rat microsatellites. Genomics, 1992, 12(4): 627-631.

BHATTARAI NR et al. PCR and direct agglutination as Leishmania infection markers among healthy Nepalese subjects living in areas endemic for Kala-Azar. Tropical Medicine and International Health, 2009, 14(4): 404-411.

. Development and evaluation of different PCR-based typing methods for discrimination of Leishmania donovani isolates from Nepal. Parasitology, 2010, 29: 1-11. 
Silva, M. A. L, Medeiros, R. A.; Filho, S. B.; Melo, L. F. Medeiros, Z./Revista Eletrônica de Farmácia Vol 7 (3), $01-15,2010$

BOTILDE $Y$ et al. Comparison of molecular markers for strain typing of Leishmania infantum. Infection, Genetics and Evolution, 2006, 6: 440-446.

BRENIĖRE, S. F. et al. Polymerase chain reaction-based identification of New World Leishmania species complexes by specific kDNA probes. Acta Tropical,1999, 73: 283-293.

BRUSTOLONI YM et al. Sensitivity and specificity of polymerase chain reaction in Giemsastained slides for diagnosis of visceral leishmaniasis in children. Memórias do Instituto Oswaldo Cruz, 2007, 102(4): 497-500.

BULLE B et al. Practical approach for typing strains of Leishmania infantum by microsatellite analysis. Journal of Clinical Microbiology, 2002, 40(9): 3391-3397.

CORTES $S$ et al. PCR as a rapid and sensitive tool in the diagnosis of human and canine leishmaniasis using Leishmania donovani s.I.-specific kinetoplastid primers. Transaction of the Royal of Society Tropical Medicine and Hygiene, 2004, 98: 12-17.

Application of kDNA as a molecular marker to analyse Leishmania infantum diversity in Portugal. Parasitology International, 2006, 55: 277-283.

CORTES SJC. Diversidade genética da população parasitária de Leishmania em Portugal. 2008. 163 p. Tese (Doutorado)-Universidade Nova de Lisboa, Lisboa.

CRUZ I et al. Spanish HIV-Leishmania study group. Nested polymerase chain reaction (Ln-PCR) for diagnosing and monitoring Leishmania infantum infection in patients co-infected with Human Immunodeficiency Virus. Transaction of the Royal of Society Tropical Medicine and Hygiene, 2002, 96(suppl. 1): 1185-1189.

Comparison of new diagnostic tools for management of pediatric Mediterranean visceral leishmaniasis. Journal of Clinical Microbiology, 2006, 44(7): 2343-2347.

DEBORGGRAEVE $\mathrm{S}$ et al. Diagnostic accuracy of a new Leishmania PCR for clinical visceral leishmaniasis in Nepal and its role in diagnosis of disease. Tropical Medicine and International Health, 2008, 13(11) 1378-1383.

DENIAU M et al. The biological diagnosis of leishmaniasis in HIV-infected patients. Annals of Tropical Medicine and Parasitology, 2003, 97(1): 15-33.

DISCH J et al. Single-step duplex KDNA-PCR for detection of Leishmania donovani complex in human peripheral blood samples. Diagnostic Microbiology and Infectious Disease, 2006, 56: 395-400.

DONCKER $S$ et al. A new PCR-ELISA for diagnosis of visceral leishmaniasis in blood of HIV-negative subjects. Transaction of the Royal of Society Tropical Medicine and Hygiene, 2005, 99: 25-31.

DOURADO ZFS et al. Panorama histórico do diagnóstico laboratorial da leishmaniose visceral até o surgimento dos testes imunocromatográficos (rk39). Revista de Patologia Tropical, Goiânia, 2007, 36(3) 205-214.

EL TAIL N et al. Genetic heterogeneity of ribosomal internal transcribed spacer in clinical samples of Leishmania donovani spotted on filter paper as revealed by single-strand conformation polymorphisms and sequencing. Transaction of the Royal of Society Tropical Medicine and Hygiene, 2000, 94: 575-579.

ELAMIN EM et al. Identification of Leishmania donovani as a cause of cutaneous leishmaniasis in Sudan. Transaction of the Royal of Society Tropical Medicine and Hygiene, 2008, 102: 54-57. 
Silva, M. A. L, Medeiros, R. A.; Filho, S. B.; Melo, L. F. Medeiros, Z./Revista Eletrônica de Farmácia Vol 7 (3), $01-15,2010$

FAKHAR $\mathrm{M}$ et al. Asymptomatic human carriers of Leishmania infantum: possible reservoirs for Mediterranean visceral leishmaniasis in southern Iran. Annals of Tropical Medicine and Parasitology, 2008, 102(7) 1-7.

FERNANDES $O$ et al. Mini-exon gene variation in human pathogenic Leishmania species. Molecular and Biochemical Parasitology, 1994, 66: 261-271.

FERREIRA ME, GRATTAPAGLIA D. Introdução ao uso de marcadores moleculares em análise genética. Brasília: Embrapa, 1998.

FISA R et al. Leishmania infantum DNA detection in urine from patients with visceral leishmaniasis and after treatment control. The American Society of Tropical Medicine and Hygiene, 2008a, 78(5): 741-744.

A nested polymerase chain reaction for diagnosis and follow-up of human visceral leishmaniasis patients using blood samples. Transaction of the Royal of Society Tropical Medicine and Hygiene, 2008b, 96(suppl. 1): 191-194.

FISSORE $C$ et al. Convenience of serum for visceral leishmaniasis diagnosis by PCR. Journal of Clinical Microbiology, 2004, 42(11) 5332-5333.

FLOETER-WINTER, LM. Descrição e utilização de alvos moleculares para identificação de Leishmania por PCR. Disponível em: <http://www.cve.saude.sp.gov.br/agencia/bepa73_leishmania.htm>. Acesso em: 3 maio 2010.

GARCIA L et al. Culture-independent species typing of neotropical Leishmania for clinical validation of a PCR-based assay targeting heat shock protein 70 genes. Journal of Clinical Microbiology, 2004, 42(5): 2294-2297.

GARCÍA-GARCÍA JA et al. Use of noninvasive markers to detect Leishmania infection in asymptomatic human immunodeficiency virus infected patients. Journal of Clinical Microbiology, 2006, 44: 4455-4458.

GAVGANI AM, KHADEMVATAN S, GHAZANCHAEI A. KAtex antigen-detection test as a diagnostic tool for latent visceral leishmaniasis cases. African J ournal of Biotechnology, 2008, 7(7): 852-859.

GERAMIZADEH B, FAKHAR M, MOTAZEDIAN MH. Visceral leishmaniasis with duodenal involvement: three immunocompetent cases from southern Iran. Annals of Tropical Medicine and Parasitology, 2006, 100: 637-640.

GONTIJO CMF, MELO MN. Leishmaniose visceral no Brasil: quadro atual, desafios e perspectivas. Revista Brasileira de Epidemiologia, 2004, 7(3): 338-349.

GUERBOUJ S et al. Gp63 gene polymorphism and population structure of Leishmania donovani complex: influence of the host selection pressure?, 2001, 122: 25-35.

HARALAMBOUS $C$ et al. Development of a molecular assay specific for the Leishmania donovani complex that discriminates L. donovani/Leishmania infantum zymodemes: a useful tool for typing MON-1. Diagnostic Microbiology and Infectious Disease, 2008, 60: 33-42.

HOYA, RD et al. Leishmania panamensis: a 44bp deletion in gp63 gene is found in cDNA and genomic libraries. Memórias do Instituto Oswaldo Cruz, 1999, 94(5): 641-643.

JUNIOR MSCL et al. Identificação de espécies de Leishmania isoladas de casos humanos em Mato Grosso do Sul por meio da reação em cadeia da polimerase. Revista da Sociedade Brasileira de Medicina Tropical, 2009, 42(3) 303-308. 
Silva, M. A. L, Medeiros, R. A.; Filho, S. B.; Melo, L. F. Medeiros, Z./Revista Eletrônica de Farmácia

Vol 7 (3), $01-15,2010$

KALLEL $\mathrm{K}$ et al. Asymptomatic bearing of Leishmania infantum among Tunisian HIV infected patients. Pathologie Biologie, 2007, 55: 521-524.

KATAKURA $\mathrm{K}$ et al. Leishmania mini-exon genes for molecular epidemiology of leishmaniasis in China and Ecuador. The Tokai Journal of Experimental and Clinical Medicine, 1999, 26(3): 393-399.

KAZEMI-RAD E et al. Diagnosis and characterization of Leishmania species in giemsa-stained slides by PCR-RFLP. Iranian J ournal of Public Health, 2008, 37(1): 54-60.

KONGKAEW $W$ et al. Autochthonous visceral leishmaniasis: a report of a second case in Thailand. Southeast Asian Journal of Tropical Medicine and Public Health, 2007, 38(1) 8-12.

KUHLS $\mathrm{K}$ et al. Multilocus microsatellite typing (MLMT) reveals genetically isolated populations between and within the main endemic regions of visceral leishmaniasis. Microbes and Infection, 2007, 9: 334-343.

KYRIAKOU DS et al. Quick detection of Leishmania in peripheral blood flow cytometry. Is prestorage leucodepletion necessary for leishmaniasis prevention in endemic areas? Transfusion Medicine, 2003, 13: 59-62.

LACHAUD L et al. Optimized PCR Using patient blood samples for diagnosis and follow-up of visceral leishmaniasis, with special reference to AIDS patients. Journal of Clinical Microbiology, 2000, 38(1): 236-240.

- Comparison of various sample preparation methods for PCR diagnosis of visceral leishmaniasis using peripheral blood. Journal of Clinical Microbiology, 2001, 39(2): 613-617.

LAMBSON B, SMYTH A, BARKER D. Sequence homology within a minicircle class of the Leishmania donovani complex. Molecular and Biochemical Parasitology, 1999, 101: 229-232.

. Leishmania donovani: Development and characterisation of a kinetoplast DNA probe and its use in the detection of parasites. Experimental Parasitology, 2000, 94: 15-22.

LE FICHOUX $Y$ et al. Occurrence of Leishmania infantum parasitemia in asymptomatic blood donors living in an area of endemicity in Southern France. Journal of Clinical Microbiology, 1999, 37(6): 1953-1957.

MAIA-ELKHOURY ANS et al. Visceral leishmaniasis in Brazil: trends and challenges. Cadernos de Saúde Pública, Rio de Janeiro, 2008, 24(12) 2941-2947.

MARFURT J et al. Identification and Differentiation of Leishmania species in clinical samples by PCR amplification of the mini-exon sequence and subsequent restriction fragment length polymorphism analysis. Journal of Clinical Microbiology, 2003a, 41(7) 3147-3153.

Diagnostic genotyping of old and new World Leishmania species by PCR-RFLP. Diagnostic Microbiology and Infectious Disease, 2003b, 46: 115-124.

MARTIN-SANCHEZ $J$ et al. Diagnosis of infections with Leishmania infantum using PCR-ELISA. Parasitology, 2001, 122: 607-615.

MARY C et al. Quantification of Leishmania infantum DNA by a real-time PCR assay with high sensitivity. J ournal of Clinical Microbiology, 2004, 42(11): 5249-5255.

. Reference values for Leishmania infantum parasitemia in different clinical presentations: quantitative polymerase chain reaction for therapeutic monitoring and patient follow-up. The American Society of Tropical Medicine and Hygiene, 2006, 75(5): 858-863. 
Silva, M. A. L, Medeiros, R. A.; Filho, S. B.; Melo, L. F. Medeiros, Z./Revista Eletrônica de Farmácia Vol 7 (3), $01-15,2010$

MATEUS RP et al. Caracterização preliminar do espaçador interno transcrito-1 its- 1 do DNA ribossômico nas espécies do Cluster buzzatii de Drosophila (Diptera: drosophilidae). Ambiência, Guarapuava, 2006, 2(1): 89-96.

MAURYA R et al. Evaluation of PCR for diagnosis of Indian kala-azar and assessment of cure. Journal of Clinical Microbiology, 2005, 43(7): 3038-3041.

MONROY-OSTRIA A, HERNANDEZ-MONTES O, BARKER DC. Aetiology of visceral leishmaniasis in México. Acta Tropical. 2000, 75: 155-161.

MORALES MA et al. Molecular tracking of infections by Leishmania infantum. Transaction of the Royal of Society Tropical Medicine and Hygiene, 2001, 95: 104-107.

Relapses versus reinfections in patients coinfected with Leishmania infantum and human immunodeficiency virus type 1. The Journal of Infectious Diseases, 2002, 185: 1533-1537.

MOTAZEDIAN $\mathrm{M}$ et al. A urine-based polymerase chain reaction method for the diagnosis of visceral leishmaniasis in immunocompetent patients. Diagnostic Microbiology and Infectious Disease, 2008, 60: 151-154.

OCHSENREITHER, S. et al. Multilocus microsatellite typing as a new tool for discrimination of Leishmania infantum MON-1 strains. Journal of Clinical Microbiology, 2006, 44(2) 495-503.

PIARROUX $\mathrm{R}$ et al. Isolation and characterization of a repetitive DNA sequence from Leishmania infantum: development of a visceral leishmaniasis polymerase chain reaction. The American Society of Tropical Medicine and Hygiene, 1993, 49(3): 364-369.

PIZZUTO $M$ et al. Role of PCR in Diagnosis and prognosis of visceral leishmaniasis in patients coinfected with human immunodeficiency virus type 1. Journal of Clinical Microbiology, 2001, 39: 357-361.

RIERA $C$ et al. Detection of Leishmania infantum cryptic infection in asymptomatic blood donors living in an endemic area (Eivissa, Balearic Islands, Spain) by different diagnostic methods. Transaction of the Royal of Society Tropical Medicine and Hygiene, 2004, 98: 102-110.

RODRIGUEZ NM et al. Genetic homogeneity within Leishmania (L.) infantum isolated from human and dogs: the relationship with the sandfly fauna distribution in endemic areas of Nueva Esparta State, Venezuela. Parasitology, 2005, 130: 611-619.

ROLÃO N et al. Quantification of Leishmania infantum parasites in tissue biopsies by real-time polymerase chain reaction and polymerase chain reaction - enzyme-linked immunosorbent assay. J ournal of Parasitology, 2004, 90(5) 1150-1154.

ROSSI $V$ et al. Structural organisation of microsatellite families in the Leishmania genome and polymorphisms at two (CA)n loci. Molecular and Biochemical Parasitology, 1994, 65(2) 271-282.

SALAM MA et al. rK39 enzyme-linked immunosorbent assay for the diagnosis of kala-azar in an endemic zone of Bangladesh. Pakistan J ournal of Medical Sciences, 2009, 25: 4635-4640.

SALOTRA P et al. Development of a species-specific PCR assay for detection of Leishmania donovani in clinical samples from patients with kala-azar and post-kala-azar dermal leishmaniasis. Journal of Clinical Microbiology, 2001, 39(3): 849-854.

SCHÖNIAN G et al. PCR diagnosis and characterization of Leishmania in local and imported clinical samples. Diagnostic Microbiology and Infectious Disease, 2003, 47: 349-358 . 
Silva, M. A. L, Medeiros, R. A.; Filho, S. B.; Melo, L. F. Medeiros, Z./Revista Eletrônica de Farmácia Vol 7 (3), $01-15,2010$

SERIDI $\mathrm{N}$ et al. Application of PCR-RFLP for the exploration of the molecular diversity of Leishmania infantum in Algeria. Transaction of the Royal of Society Tropical Medicine and Hygiene, 2008, 102: 556-563.

SERIN MS et al. Rapid diagnosis and genotyping of Leishmania isolates from cutaneous and visceral leishmaniasis by microcapillary cultivation and polymerase chain reaction-restriction fragment length polymorphism of mini-exon region. Diagnostic Microbiology and Infectious Disease, 2005, 53: 209-214.

SHARMA $\mathrm{P}$ et al. Comparative in vivo expression of amastigote up regulated Leishmania genes in three different forms of leishmaniasis. Parasitology International, 2010, 59(2): 262-264.

SMYTH AJ et al. Rapid and sensitive detection of Leishmania kinetoplast DNA from spleen and blood samples of kala-azar patients. Parasitology, 1992, 105: 183-192.

SREENIVAS G et al. Nested PCR assay for detection of Leishmania donovani in slit aspirates from post-kala-azar dermal leishmaniasis lesions. Journal of Clinical Microbiology, 2004, 42(4): 17771778.

STARK D et al. Post-kala-azar dermal leishmaniasis due to Leishmania infantum in a human immunodeficiency virus type 1-infected patient. Journal of Clinical Microbiology, 2006, 44(3): 11781180.

SUKMEE $\mathrm{T}$ et al. A suspected new species of Leishmania, the causative agent of visceral leishmaniasis in a Thai patient. International Journal of Parasitology, 2008, 38: 617-622.

SUNDAR S, RAI M. Laboratory diagnosis of visceral leishmaniasis. Clinical and Diagnostic Laboratory Immunology. 2002, 9: 951-958.

TELLERIA J et al. Trypanosoma cruzi: sequence analysis of the variable region of kinetoplast minicircles. Experimental Parasitology, 2006, 114(4): 279-288.

TUPPERWAR AN et al. Development of a real-time polymerase chain reaction assay for the quantification of Leishmania species and the monitoring of systemic distribution of the pathogen. Diagnostic Microbiology and Infectious Disease, 2008, 61: 23-30.

ULIANA SR et al. Leishmania: genus identification based on a specific sequence of the $18 \mathrm{~S}$ ribosomal RNA sequence. Experimental Parasitology, 1991, 72: 157-163.

VAN EYS GJJM et al. Sequence analysis of small subunit ribosomal RNA genes and its use for detection and identification of Leishmania parasites. Molecular and Biochemical Parasitology, 1992, 51: 133-142.

VILLINSKIA JT et al. Evidence for a new species of Leishmania associated with a focal disease outbreak in Ghana. Diagnostic Microbiology and Infectious Disease, 2008, 60: 323-327.

WALLACE GR et al. Mapping of a visceral leishmaniasis-specific immunodominant B-cell epitope of Leishmania donovani Hsp70. Infection and Immunity, 1992, 60: 2688-2693. 